\title{
A Contribution to the Simulation of the Process of Fire Detection
}

\author{
CLAUDIA REXFORT \\ Department of Communication Systems \\ University Duisburg-Essen \\ Duisburg, Germany
}

\begin{abstract}
The combination of a fire model and a smoke sensor model to simulate the response of the sensor to different fire situations is presented. The Fire Dynamics Simulator (FDS) of the National Institute of Standards and Technology was combined with a smoke sensor model developed at the Institute of Communication Systems of the University DuisburgEssen. The simulation results of the smoke development during a fire were converted into input parameters suitable for the smoke sensor model. To simulate a smoke sensor several input parameters are necessary to describe the smoke, the sensor, and its housing. The size distribution of the smoke particles is one important parameter to describe the smoke. Coagulation of the smoke particles that influences the particle size distribution is implemented in the model. Simulation results of the combined model are compared to measurement results of an open flaming fire and a smoldering fire. A scattering light sensor and an ionization chamber were simulated. The results of measurements and simulations show that the quality of the sensor simulation depends on the fuel that is burned.
\end{abstract}

KEYWORDS: modeling, smoke, detection

\section{NOMENCLATURE}

$\begin{array}{llll}d_{g} & \text { geometric mean diameter } & v_{g, 0} & \text { initial geom. mean volume } \\ i_{c} & \text { chamber current } & \text { Greek } & \\ I_{0} & \text { emitted intensity } & \beta(u, v) & \text { coagulation rate } \\ I_{S} & \text { scattered intensity } & \lambda & \text { wavelength of light } \\ k & \text { Boltzman constant } & \mu & \text { dynamic viscosity } \\ K_{c} & \text { coagulation coefficient } & \rho_{\text {smoke }} & \text { smoke mass density } \\ n(v, t) & \text { particle size distribution } & \rho_{\text {part }} & \text { specific smoke mass density } \\ N & \text { particle number concentration } & & \\ N_{o} & \text { initial particle number } & \ln \sigma & \text { standard deviation } \\ & \text { concentration } & & \\ T & \text { temperature } & \ln \sigma_{0} & \text { initial standard deviation }\end{array}$

$v_{g} \quad$ geometric mean volume

\section{INTRODUCTION}

Computer simulations are becoming more and more important in various fields. They help to understand and optimize different kinds of processes. They are also an important tool in fire detection simulations. Simulations give a better and more detailed understanding of the physics and chemistry of combustion. Fire sensor models give the opportunity to verify the signals measured by a fire sensor to decide which part of the signal is caused by the fire and which part of the signal is caused by the sensor or the 
environment of the fire. With fire simulations it is possible to define the burning material and the environment of the fire more precisely than during a fire experiment, so that one knows their properties. A combination of a fire model and a fire sensor model will give the opportunity to investigate the influence of different parameters of the fire and its environment on the signal obtained by the fire sensor. In the Fire Dynamics Simulator [10] some properties of detectors are implemented. For instance, the entry time lag of smoke intruding the housing of the detector is considered, but the sensors themselves are not modeled in detail.

\section{SENSOR MODEL}

At the Institute of Communication Systems of the University Duisburg-Essen, a general model of a fire sensor in its housing has been developed by Gockel [4]. The model output provides the sensor response to a given fire or non-fire situation. The fire situation and the fire sensor are defined by the input parameters of the model. Different types of sensors can be simulated with the sensor model, e.g., different types of smoke sensors, heat sensors and some gas sensors. The focus of this paper is on the smoke sensor models.

Figure 1 shows the structure of the sensor model. The sensor model mainly consists of four parts: the housing, the dynamic effects of the housing, the sensor and the effects of the electronic named A, B, C, D respectively. These parts represent different physical parts and/or properties of the sensor.

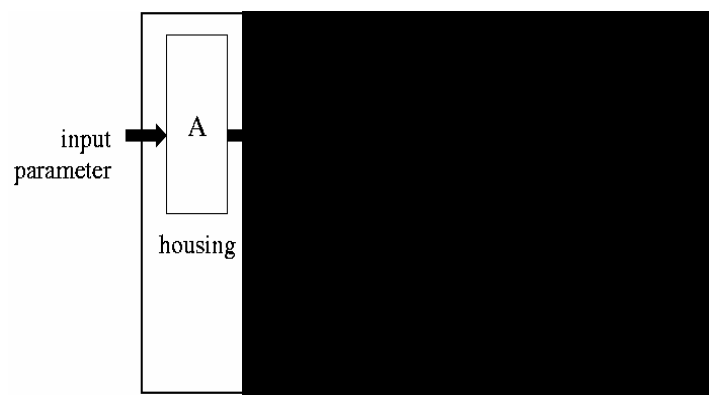

Fig. 1. Model of a fire sensor in its housing [3].

The model part A is a memory-less system that represents the housing of the sensor and its time invariant properties. The housing of the sensor acts like a band-pass filter on the smoke particles. Different processes filter out the large particles, e.g., gravitational settling, while other processes filter out the small particles, e.g., electrostatic attraction or diffusional losses to the walls of the housing. Thus the largest and the smallest particles of the smoke are filtered out. The different effects are mostly time independent.

Part B of the model describes the entry-lag for smoke particles intruding the housing. Heskestad [5] describes the housing as a time delaying system of first order, where an impulse response can be defined for the housing. Part B is divided in two parts, B1 and B2. Part B2 represents a linear time invariant system, which convolutes every parameter transmitted from model part A with an impulse response [4]. The impulse response is provided by model part B1. This impulse response depends on the velocity of the incoming flow, and is thus time dependent. In Cleary et al. [3] results of investigations of the parameters of the impulse response are given. The combination of model parts B1 and B2 form a time variant system of first order. 
Part $\mathrm{C}$ is a realization of the sensor characteristics, i.e., the conversion from the measured values to the sensor signal. Part $\mathrm{C}$ of the model is described in more detail for a photoelectric sensor and an ionization chamber below.

Part D represents possible effects of the electronics at the output side of the sensor, e.g., low-pass effects or noise of the electronic circuits.

\section{SMOKE MODEL}

To simulate smoke sensors a model that describes the main properties of the smoke has to be used. Gockel's model is based on a simple smoke model, which describes the two main properties of the smoke affecting smoke detection for light scattering detector and ionization chamber sensors [4].

One main property that describes smoke is the size distribution of the smoke particles. To describe the smoke by the size distribution of its particles a spherical shape is assumed. The particle size distribution $n(v, t)$ is given by the logarithmic normal distribution [7], where the logarithm of the particle size is normally distributed.

$n(v, t)=\frac{N}{3 \sqrt{2 \pi} \ln (\sigma) v} \exp \left(\frac{\ln ^{2}(v / v g)}{18 \ln \sigma^{2}}\right)$

$N$ is the particle number concentration, $v_{g}$ is the geometric mean particle volume, and $\ln \sigma$ is the standard deviation of the distribution.

Another characteristic property of the smoke is its absorption of light. This is described by the complex refractive index of the smoke. The refractive index is complicated to measure, so for the smoke model it is represented by the carbon fraction of the smoke. To include this property of smoke in the model Gockel assumes that the smoke particles only consist of carbon in its pure or in bounded form, e.g., $\mathrm{CO}_{2}$ and CO. With this approximation the degree of absorption of light by the smoke can be defined by the carbon fraction, whereby carbon is the only material emerging from a fire that absorbs visible or infrared light to a high degree [1]. The larger the carbon fraction is, the larger is the absorption of light. Consequently the carbon fraction is a possible parameter to model optical procedures of smoke measurement.

\section{SENSOR PRINCIPLES}

\section{Scattering Light Sensors}

Scattering light sensors use the effect of scattering of light by smoke particles to measure the amount of smoke. Therefore light is described as an electromagnetic wave. The sensor measures the intensity of the scattered light (scattered light sensors), when the electromagnetic wave meets a particle. In this case the electric and magnetic fields have to fulfill Maxwell's Law inside the particle and outside the particle as well as the boundary conditions. In a mathematical view a boundary value problem on a sphere has to be solved. The solution of this boundary value problem is based on a calculation done 
by Mie and is therefore called the 'Mie Theory.' The intensity $I_{\mathrm{s}}$ of the scattered light depending on the incoming light $I_{0}$ can be described as [4]

$$
I_{s}=I_{0} \frac{\lambda^{2}}{8 \pi^{2} a^{2}}\left(i_{1}(\alpha, \underline{m}, \Theta)+i_{2}(\alpha, \underline{m}, \Theta)\right)
$$

following the Mie Theory. $\lambda$ is the wavelength of the incoming light and $a$ the distance between the scattering particle and the light receiver. $i_{1}$ and $i_{2}$ are two intensity functions, which depend on the ratio between the particle radius and the wavelength of light $\alpha$, on the complex refractive index $\underline{m}$ and on the scattering angle $\Theta$. The intensity of the scattered light is proportional to the number of particles. In general only the number concentration of particles is known. So the intensity of light scattered by one particle has to be multiplied by the number concentration $N$ and the scattering volume $V_{S C}$. The intensity of light scattered by particles of the same size becomes

$$
I_{S}=I_{0} \int_{0}^{\infty} \frac{N \cdot V_{s c} \lambda^{2}}{8 \pi^{2} a^{2}}\left(i_{1}(\alpha, \underline{m}, \Theta)+i_{2}(\alpha, \underline{m}, \Theta)\right) n(d) \boldsymbol{d} d
$$

The $n(d)$ is the diameter distribution of the particles.

\section{Ionization Chamber}

Smoke sensors that are based on the fact that ions of air agglomerate on the surfaces of smoke particles [4], are called ionization chambers. The ionization chamber consists basically of the chamber volume with two electrodes, which form a plate capacitor. At one of these electrodes a radioactive substrate is mounted which produces ions in the air volume between the electrodes. A dc voltage is applied to the electrode and due to the ions there is a current inside the capacitor. If particles intrude into the chamber volume the ions accumulate on the surfaces of these particles. Due to their adhesion at largersized smoke particles the ions are of lower mobility, cannot flow to the plates, thus so the current decreases. The decrease of the current, often called chamber current, is a measure for the number of particles inside the plate capacitor [6]. The chamber current without intruding smoke particles is denoted as $i_{0}$. Then the relation between the particle number and size and the chamber current $i_{c}$ inside the capacitor is [4] for $n$ smoke particles with diameter $d$.

$$
d \cdot n=\eta\left(\frac{i_{0}}{i_{C}}-\frac{i_{C}}{i_{0}}\right)
$$

This equation is called the characteristic chamber equation, with $\eta$ the chamber constant, which is mainly determined by the geometry of the chamber. If the particles have different sizes, the particle size distribution has to be taken into account in the characteristic chamber equation:

$$
\int_{0}^{\infty} d \cdot n(d) \mathrm{d} d=\eta y
$$


For the simulation of the two sensors the model needs the following input parameters:

- particle number concentration $N$,

- geometric mean particle diameter $d_{g}$ (volume $v_{g}$ ),

- standard deviation of the size distribution $\ln \sigma$, and

- carbon fraction (optical sensor).

\section{PARTICLE NUMBER CONCENTRATION}

The particle number concentration of the smoke shall be computed from the results given by a fire simulation. For the fire simulations the Fire Dynamics Simulator (FDS) developed at the National Institute of Standards and Technology, Maryland USA [10], is used. In simulating the amount of smoke produced by the fire FDS gives the smoke mass density as a result. From this smoke mass density $\rho_{\text {smoke }}$ the parameters of the particle size distribution have to be calculated. The relation between the particle size distribution and the smoke mass density is then given by:

$N_{0}=\frac{\rho_{\text {smoke }}}{\rho_{\text {part }}} v_{g}^{-1} \exp \left(-\frac{9}{2} \ln ^{2} \sigma\right)$

$\rho_{\text {part }}$ is the specific smoke mass density and $\rho_{\text {smoke }}$ is the smoke mass density calculated with the fire model. This equation can be obtained from the first order Moment of the lognormal size distribution [8]. Using this equation the particle number concentration can be calculated from the smoke mass density.

\section{Implementation of Coagulation}

The process of coagulation influences the size distribution of the particles. Two colliding particles coagulate to one particle, which decreases the number of particles and increases the size of the particles but has no effect on the total mass of the particles. The effect of coagulation can be described by the following equation [7].

$\frac{\partial}{\partial t} n(v, t)=\frac{1}{2} \int_{0}^{v} \beta(u, v-u) n(u, t) n(v-u, t) d u-n(v, t) \int_{0}^{\infty} \beta(u, v) n(u, t) d u$

$\beta(u, v)$ is the so-called coagulation kernel.

Assuming that the size distribution stays lognormal and the standard deviation of the distribution does not change much a solution for this equation can be found using the moments method [4].

$$
\frac{N(t)}{N_{0}}=\frac{1}{1+\left[1+\exp \left(\ln ^{2} \sigma_{0}\right)\right] K_{c} N_{0} t} .
$$


$K_{c}=2 k T /(3 \mu)$ is the collision coefficient which can be calculated with the temperature $T$ and the dynamic viscosity $\mu$, that FDS provides. Also results for the effect of coagulation on the geometric particle volume and the standard deviation of the distribution can be given as [7].

$$
\frac{v_{g}(t)}{v_{0}}=\frac{\exp \left(9 \ln ^{2}\left(\sigma_{0}\right) / 2\right) \cdot\left[1+\left\{1+\exp \left(\ln ^{2} \sigma_{0}\right)\right\} K_{C} N_{0} t\right]^{3 / 2}}{\left[2\left(1+\left\{1+\exp \left(\ln ^{2} \sigma_{0}\right)\right\} K_{C} N_{0} t\right)+\exp \left(9 \ln ^{2} \sigma_{0}\right)-2\right]^{1 / 2}}
$$

$v_{g, 0}=v(0)$ is the initial geometric mean particle volume for the lognormal distribution. It is assumed that the particle sizes stay log-normally distributed but the geometric standard deviation of the size distribution changes due to coagulation.

$$
\ln \sigma(t)=\frac{1}{9} \ln \left(2+\frac{\exp \left(9 \ln ^{2} \sigma_{0}\right)-2}{1+\left[1+\exp \left(\ln ^{2} \sigma_{0}\right)\right] K_{C} N_{0} t}\right)
$$

Figure 2 shows a comparison of the particle number concentration measured during an EN54 n-heptane (TF5) testfire experiment [2] and two simulated particle number concentrations, one with coagulation and one without. The testfire has been ignited at $2 \mathrm{~s}$ and burns out at $200 \mathrm{~s}$.

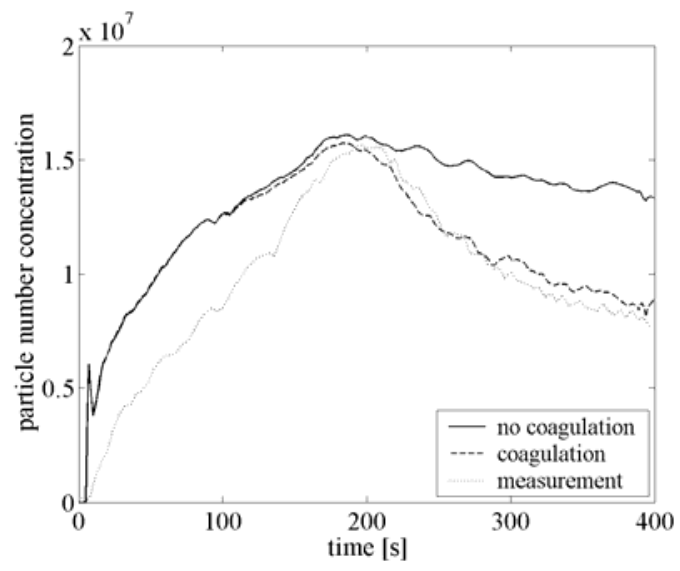

Fig. 2. Particle number concentration (n-heptane fire).

At the beginning of the fire the simulated signals showed a jump while the measured one increased nearly linearly. The coagulation started to effect the simulation at about $100 \mathrm{~s}$ after the fire had been ignited. At this point of time the number of particles reached a value at which coagulation started to become recognizable. After the fire was extinguished, the measured particle number concentrations and the one simulated with coagulation started to decrease fast, while the simulation without coagulation decreased much slower. This comparison shows, that the coagulation has to be taken into account to get suitable simulation results.

It has to be stated that the measured results are influenced by the measurement procedure. During the measurement the smoke is sucked through a tube, diluted by clean air and 
than the number of particles is measured [9]. This is a reason for the difference between the simulated and measured signals in the beginning of the fire. Observing the fire experiment and the fire simulation there is a cloud of smoke arising as soon as the nheptane is ignited, this explains the jump like increase of the simulated signals. Comparing the visual observation of the experiment with the results shown in Fig. 2, the simulation results seem to be more reasonable.

\section{RESULTS}

In this chapter results of the simulation of two types of smoke detectors are shown, a scattered light sensor and an ionization chamber. The data are measured during two EN54 testfire experiments, the n-heptane testfire (TF5) and the smoldering cotton wick testfire (TF3), with a common scattered light sensor [12] and a measurement ionization chamber [11] used for reference measurements during the fire experiments. The sensor signals were measured in the fire detection laboratory of the University Duisburg-Essen at a radius of $3 \mathrm{~m}$ around the fireplace under the ceiling with a ceiling height of $4 \mathrm{~m}$. For the $\mathrm{n}$-heptane fire, $650 \mathrm{~g}$ n-heptane was ignited in a metal basin with an area of $1100 \mathrm{~cm}^{2}$. For the cotton wick fire 90 cotton wicks were fixed on a mounting in shape of a ring with a diameter of $10 \mathrm{~cm}$ so that they form a chimney to lead the smoke. Then the cotton wicks were ignited and the flames were immediately extinguished so that the wicks smolder but did not burn with open flame. For the FDS simulations the fire laboratory has been modeled regarding its physical parameters. To reduce the number of cells in the computational grid, a denser grid has been used in the region of the fire and larger gird cells at the region further away from the fire. Because it is impossible to model every cotton wick due to the resolution of the grid structure, the cotton wicks were modeled using a chimney shaped obstacle with the material properties of the cotton wicks. The simulation results were taken at a discrete position in computational domain regarding the fire experiments.

To verify the results, three different signals are shown in the diagrams. The first one is a signal measured during a fire experiment (measurement), the second signal is a simulation of the smoke sensor with the input parameters measured during the same fire experiment (sensor simulation) and the third signal is a simulation of the sensor using simulated inputs (over-all simulation).

\section{Simulation of Scattered Light Sensor}

Figure 3 shows the simulated and measured weighted scattered intensity. The scattered light sensor is sensitive to small changes in the input parameters, for it measures the intensity of the light that is scattered in a defined direction, which is a very small part of the incoming light. This way even small changes in the particle number or size have strong effects on the sensor signal. 


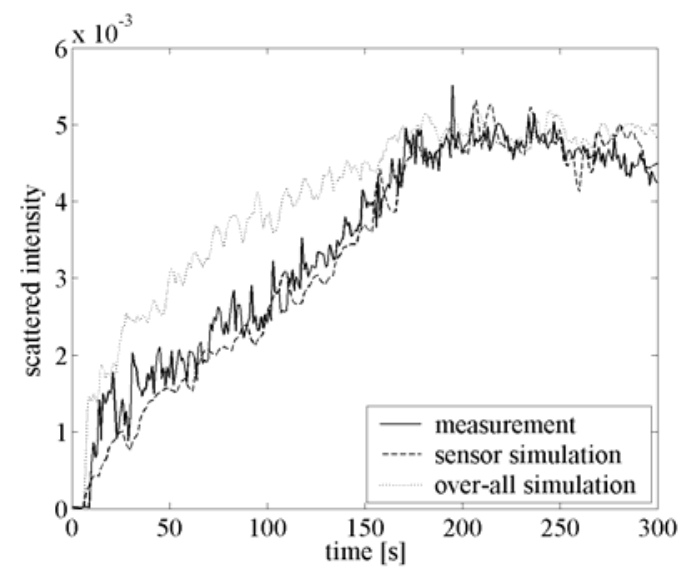

Fig. 3. Scattered light sensor simulation for an n-heptane fire.

After the fuel was ignited at about $6 \mathrm{~s}$ the measured and the overall simulated signal showed a strong increase. $30 \mathrm{~s}$ after the fire has been ignited the measured and the sensor simulated signals showed a similar nearly linear progression, while the overall simulated signal had a stronger increase in the beginning and than the degree of increase reduces. After $270 \mathrm{~s}$ all three signals reached nearly the same value. The difference in the progression of the sensor simulated signal and the over-all simulation has already been seen in the particle number concentration, see Fig. 2. The progression of the simulated signals follows the progression of the particle number concentration used as input parameters, see Fig. 2. The sensor model assumes particles of a spherical shape, though during an n-heptane fire soot agglomerates of a more complex shape are built. The results suggest that the assumption that the particles are spherical is not completely suitable for soot particles.

Figure 4 shows the signals for a smoldering fire. It can be seen that all three signals show a high fluctuation. Due to the low energy release of the smoldering cotton wicks, there was just a very small thermal flow, which transported the smoke. There was no constant stream of smoke arising like that from the n-heptane fire, and the reproducibility of the experiment was not good. But the two simulated signals show a good correlation with the measured signal. Smoldering fires produce larger, spherical particles as opposed to the small soot agglomerates produced by open flaming fires, so the assumption of spherical particle in the model does not represent a problem for smoldering fires. In general optical sensors are more suitable for smoldering fires than for open flaming fires. 


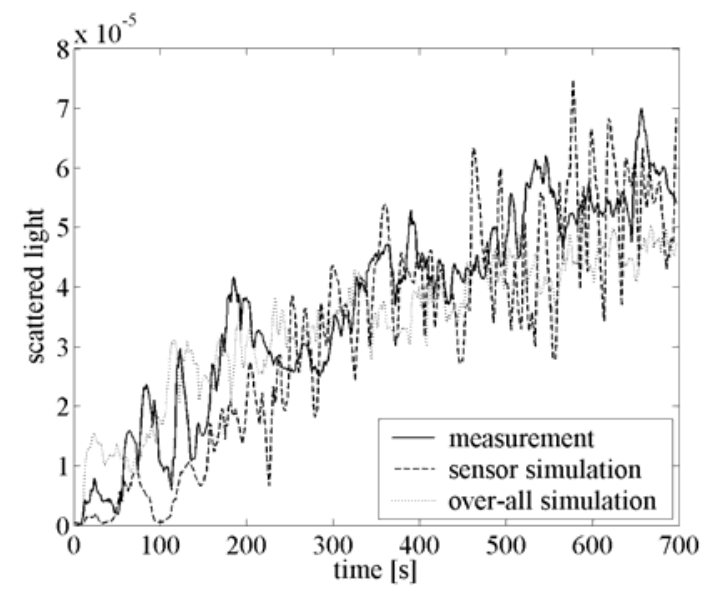

Fig. 4. Scattered light simulation for a cotton wick fire.

\section{Simulation of an Ionization Chamber}

Figure 5 shows the weighted chamber current of the ionization chamber. For the ionization chamber the measured and the overall simulated signal showed a sudden decrease at the beginning of the fire. They show a good correlation for the entire progression of the fire. While the signal sensor simulated signal with measured input parameters showed a different progression.

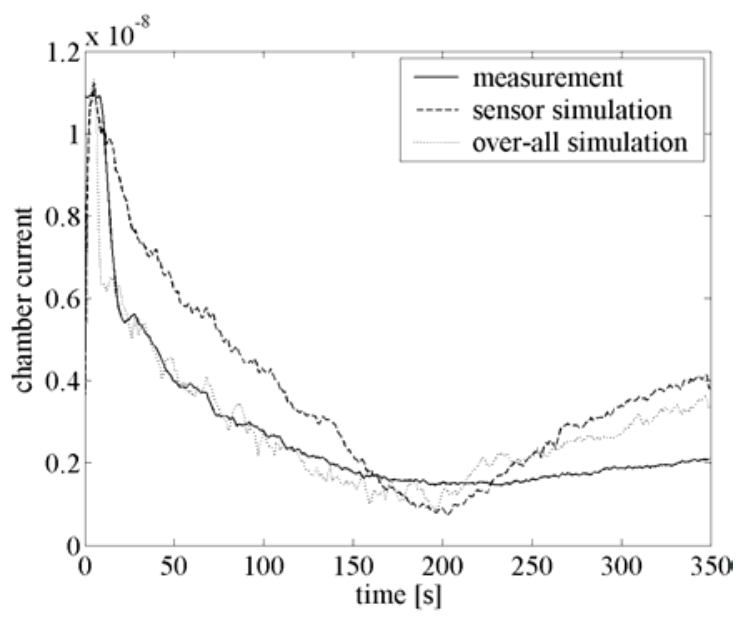

Fig. 5. Chamber current simulation for an n-heptane fire.

Compared to measurement results the overall simulation gave better results for the ionization chamber then the sensor simulation. Which suggest that the simulated particle number concentration complied the actual particle number concentration better than the measurement results, which are affected by the measuring procedure. The ionization chamber is more suitable for small soot particles than optical measurement procedures. For their simulation the assumption of spherical particles has no negative effect on the simulation results. 
For the cotton wick fire, see Fig. 6, both simulated signals differed from the measured one. The measured chamber current decreased stronger than the simulated ones. For the ionization chamber, the sensor simulation of open flaming fires like the n-heptane fire gives better results compared to measurement than the simulation of smoldering fires. The ionization chamber is more sensitive to small particles, while smoldering fires produce large particles.

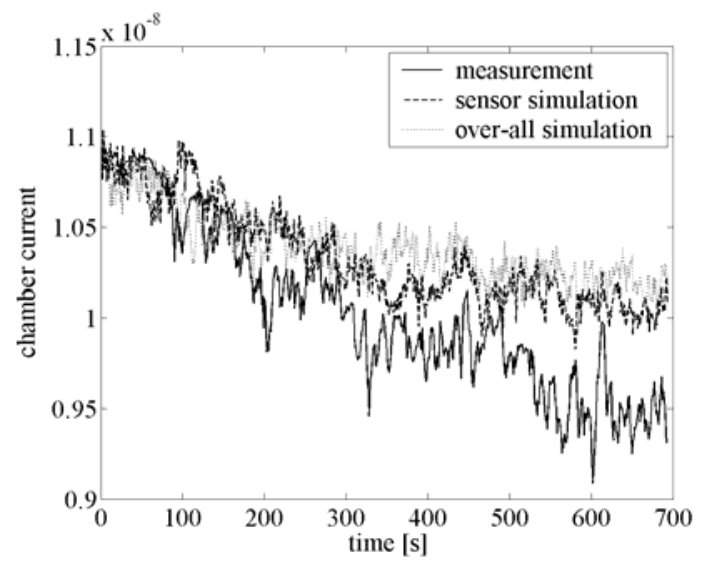

Fig. 6. Chamber current simulation for a cotton wick fire.

\section{CONCLUSION}

A combination of a fire model and a smoke sensor model was introduced. The input parameters of the smoke sensor model were obtained from results of the fire model. The effect of coagulation of the particle size distribution was implemented in the post processing of the results of the fire simulation. The simulation results of the fire and firesensor model gave good results compared to measurements. The presented results show that the quality of the simulation of different smoke sensors depend on the kind of fire (open flaming or smoldering) that is modeled. The developed model gives the opportunity to simulate different smoke sensors from the fire up to the output signal of the smoke sensors. Contrary to measurements, the simulations allow investigating the influence of a single environmental parameter on the sensor output. The presented combined model of a fire and a smoke sensor is a first part of a model of the automatic fire detection, which gives the opportunity to investigate the influence of the fire, its environment and the fire sensor on the process of fire detection.

\section{REFERENCES}

[1] Bohren, C.F. and Huffman, D.R., Absorption and Scattering of Light by Small Particles, John Wiley \& Sons, USA, 1983.

[2] CEN - The European Committee for Standardization: EN54 part9, Bestandteile automatischer Brandmeldeanlagen, Erprobungstest, 1984.

[3] Cleary, Th., Chernovski, A., Grosshandler, W., and Anderson M., "Particulate Entry Lag in Spot-Type smoke Detectors," Proceedings of the Sixth International Symposium on Fire Safety Science, Pointiers, 1999. 
[4] Gockel, F., Ein allgemeines Modell für Brandsensoren im Gehäuse, PhD Thesis, University-Duisburg Essen, Duisburg, Germany, 2001.

[5] Heskestad, G., "Generalized Characterization of Smoke Entry and Response for Products-of-Combustion Detectors," Proceedings AUBE'75, Aachen, 1975, pp. 267-310.

[6] Hosemann, J.P., Gilson, H., Messkammer nach dem Kleinionenanlagerungsprinzip zum quantitativen Nachweis von Aerosolpartikeln, Forschungsbereicht des Landes Nordrhein-Westfalen Nr. 2336, Westdeuetscher Verlag Opladen, 1973.

[7] Lee, K.W., "Change of Particle Size Distribution during Brownian Coagulation,” Journal of Colloid and Interface Science, 92: pp. 315-325, 1983.

[8] Rexfort, C., A Contribution to Fire Detection Modelling and Simulation, PhDThesis, University-Duisburg Essen, Duisburg, Germany, 2004.

[9] Tamm, E., Mirme, A., Sievert U., and Franken D., "Aerosol Particle Concentration and Size Distribution Measurements of Test-Fires as Background for Fire Detection Modeling,” Proceedings AUBE'99, Universität DuisburgEssen, Germany, 1999.

[10] Fire Dynamics Simulator - Technical Reference Guide, NIST, Gaithersburg, USA, 2001.

[11] CERBERUS LTD.: Messionisationskammer MIC, Technische Beschreibung, CERBERUS LTD., Männedorf, 1991.

[12] Siemens AG: BR82 Rauchmelder, Thilo P., Post O., Luck H., Siebel R., Klose J., Schlußbericht, Gemeinschaftliches Projekt der Siemens AG München und der Universität-Gesamthochschule-Duisburg, Germany, 1989. 
